\title{
CRISIS VITAL DESDE UNA PERSPECTIVA DE GÉNERO VITAL GRISIS FROM A GENDER POINT OF VIEW
}

\author{
Olga Flores y Eva Castro \\ Servicio de Psicología Aplicada
}

Recibido 05-02-04

Aceptado 12-04-04

\section{Resumen}

A continuación se presenta el caso de una mujer de 55 años que presenta una crisis de identidad, abordado desde una perspectiva de género y un modelo explicativo y terapéutico que incluye elementos del análisis transaccional y la terapia constructivista
Palabras clave: Crisis de identidad, terapia constructivista, perspectiva de género

\begin{abstract}
A case study of a 55 year-old-woman suffering from a vital crisis is presented, from a gender point of view and a theoretical model that includes elements from the transactional analysis and the constructivist therapy.
\end{abstract}

Key words: Vital crisi, constructivist therapy, gender perspective. 


\section{Introducción}

En el momento en que vivimos, los roles de género están sufriendo transformaciones en muy pocos años. Hace tan sólo tres décadas, la mujer se veía privada de derechos legales como heredar o trabajar sin necesidad del consentimiento de un varón. Estos cambios en la legislación se han visto acompañados, aunque no en la misma medida, de cambios en las actitudes y en las identidades establecidas socialmente para cada género.

En el presente trabajo, se plantea el caso de una paciente de 55 años que sufre una crisis vital.

Para comprender este proceso, el abordaje se hace desde una perspectiva de género e incluyendo elementos del análisis transaccional y la terapia constructivista.

Respecto al primer enfoque, para Amorós (1995), "el proceso de socialización implica que cada género tiene que haber interiorizado las pautas necesarias para saber qué tiene que pensar o hacer para satisfacer las expectativas de género". Este proceso de interiorización se produce en cuatro contextos de influencia que afectan al desarrollo del niño/a (Bronfenbrenner, 1979):

- Microsistema: la familia.

- Mesosistema: entornos cercanos (centros de salud y educativos).

- Exosistema: ambientes sociales, empleo y medios de comunicación.

- Macrosistema: valores culturales, creencias, sucesos históricos y sistema económico.

Los valores y creencias determinados por el sistema económico se reproducen en el mesosistema y en el exosistema a través de los estereotipos y roles, que, a su vez, son asimilados y transmitidos en el microsistema (la familia) a través de mensajes y guiones de vida.

Por otra parte, los rasgos que definen la identidad femenina en una sociedad patriarcal (Amorós, 1995 y Murillo, 1996; Tannen, 1996) son los siguientes:

\section{- negación de la autonomía}

- identidad supeditada a la del varón, identidad defectiva del varón
- sumisión a la autoridad masculina

- reducción de la identidad a la vida doméstica - negación de las vidas privada y publica

- identificación "ser buena persona - ser buena madre - ajustarse a las expectativas del rol de género"

- moral del cuidado: sensibilidad, empatía, actitudes de cuidado hacia la pareja e hijos/as, disposición a perdonar, comprender y liberar de sus cargas a los hombres

- posponer o negar los propios intereses y deseos, de un modo esencial

- responsabilidad, no hacer daño (aplicado a los demás; si estos conceptos se aplican a sí misma, tanto la propia mujer como los demás calificarán este comportamiento de egoísta)

- dar más de lo que se recibe

Dentro de las distintas modalidades de las sociedades patriarcales, destacamos el patriar. cado de consentimiento, en el que la mujer cree obrar en libertad y asume el rol descrito anteriormente.

\section{Consecuencias de la asunción o el rechazo del rol de género}

Desde la perspectiva del análisis transaccional, podemos establecer que la asunción versus el rechazo del rol establecido socialmente conllevan una serie de consecuencias anticipadas (positivas o negativas respectivamente), que facilitan la "elección" de la primera opción.

En el caso de asumir esta identidad, se prevé que se recibirán "bienes", según Kertesz y Induni (1978):

- Tiempo

- Caricias positivas (afecto)

- Información útil

- Bienes materiales

En el caso de que la persona no se ajuste a dicho estereotipo, existen una serie de medios para motivarla a hacerlo sin conciencia de su Adulto:

Por temor, tanto al rechazo social como de la propia pareja en el caso de que no se cumpla 
esta identidad, aspecto reforzado por la relación de poder-dependencia que se establecerá entre hombres y mujeres en una sociedad patriarcal.

Por culpa, generada al asociarse socialmente los rasgos citados anteriormente con el constructo "ser una mala persona/mujer"

Por soborno, estableciendo únicamente caricias condicionales, aquellas otorgadas si la persona manifiesta una actitud o un comportamiento aprobados socialmente.

A través de descalificaciones, que suponen una respuesta que cierra los cauces de comunicación. Se niega la existencia del otro (recordamos que uno de los rasgos del rol femenino en una sociedad patriarcal es la supeditación de su identidad a la del varón). Las formas de usar la descalificación ante un estímulo, según Berne (1981) son:

- Descalificar el propio estímulo.

- Descalificar la importancia de un problema.

- Descalificar la forma de resolución.

- Descalificar la propia capacidad para resolver el problema.

Mientras la persona acepte el rol, se producirán transacciones complementarias, fundamentalmente del tipo Niña Adaptada (NA) Sumisa - Padre Crítico, a través de las cuales recibe caricias, que, aunque dolorosas externamente, producen bienestar interno (suponen un reconocimiento).

Las caricias que predominan en esta categoría de transacciones son de tipo "lastimero", que fomentan el desvalimiento, la culpa, la disminución de la autoestima y motivan el crecimiento perjudicial del NA sumiso. Ejemplos de este tipo de caricias son "Mira lo que me has hecho hacer", cuyo componente principal es la culpabilización y hacer responsable al otro de los propios actos, o "Si no fuera por ti", actitud de reproche, con la que se responsabiliza de las limitaciones propias o la no realización de deseos al otro.

La posición existencial que mejor se ajusta al rol tradicional femenino es "Yo estoy mal - Tú estás bien", en la que la persona toma para sí lo negativo y proyecta en lo exterior lo positivo. Como vivencia que todo lo bueno está fuera de sí misma, las caricias positivas, aunque deseadas, son rechazadas, recogiendo aquellas caricias agresivas o de lástima que refuerzan su posición existencial. El correlato emocional son los sentimientos de inferioridad y el estado depresivo.

En este sentido, se mencionan a continuación las "Leyes de la economía de caricias" (Steiner, 1981), que constituyen las reglas parentales (producen sensación NO OK - no bienestar-) aceptadas socialmente que regulan su intercambio. Esquemáticamente son:

- No dé las caricias positivas que corresponda dar.

- No acepte las caricias positivas que merezca.

- No pida las caricias que necesita.

- No se dé a sí mismo caricias positivas.

- No rechace caricias negativas.

Estas leyes provocan una carencia de caricias positivas y un déficit en la salud emocional.

\section{Motivo de consulta}

J. es una mujer de 55 años, casada y con dos hijos, uno de los cuales convive aún con la pareja. Durante varios años trabajó de forma remunerada, situación que interrumpió tras el matrimonio. La demanda fundamental que plantea es "saber quién soy" y "tener más seguridad ante R." (pareja). En el momento de comenzar la terapia presenta un bajo estado de ánimo y altos niveles de ansiedad, precipitados por una crisis de pareja.

\section{Comprensión del problema}

\section{Instrumentos de evaluación empleados}

- Entrevista

- SCL-90-R

- B.D.I. Puntuación directa: 20. Depresión moderada.

- S.T.A.I.

- Pc Estado: 70.

- PC Rasgo: 60. 


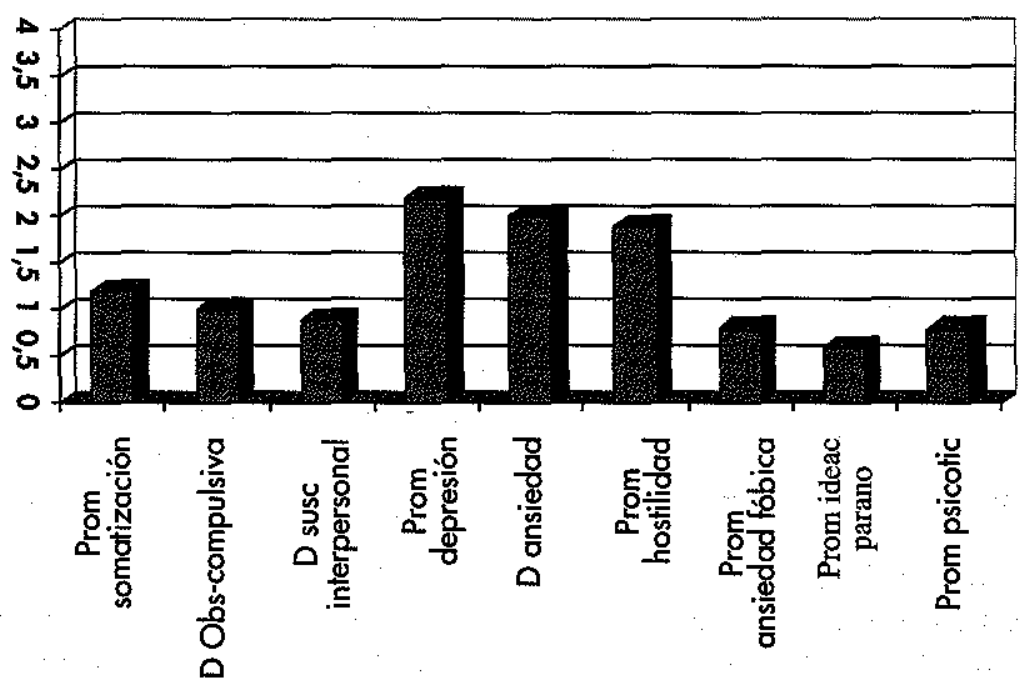

Gráfico 1. Resultados pre SCL-90

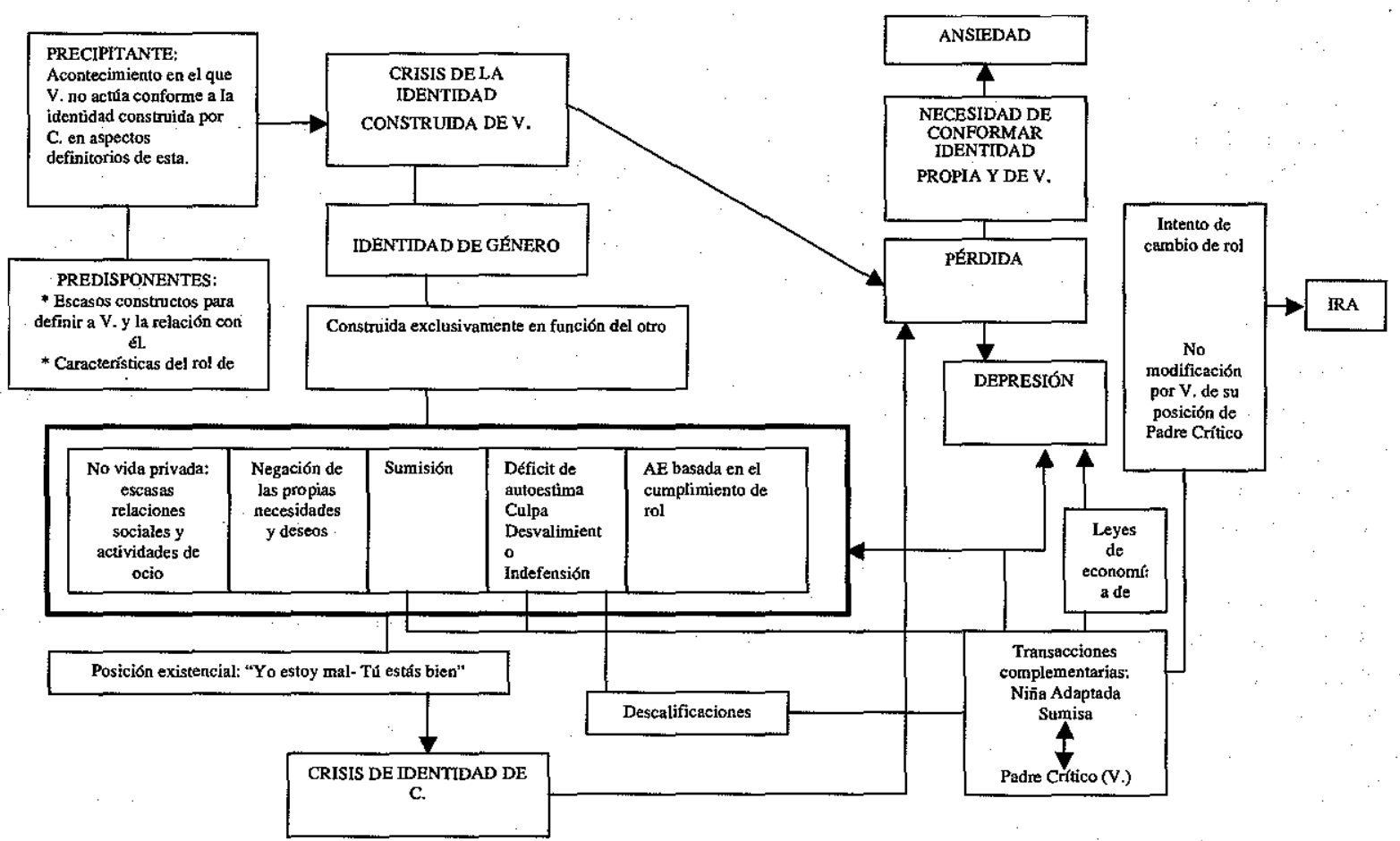

Figura 1. Modelo funcional del caso 


\section{Modelo funcional}

La crisis de identidad de J. es precipitada, a su vez, por la crisis de la identidad construida de R. (pareja). Para establecer esta relación se hace imprescindible tener en consideración uno de los rasgos fundamentales del rol de género: la construcción de la identidad femenina en función de la pareja. Otros rasgos de esta son los descritos en la introducción teórica, que aparecen también en J. La actitud de sumisión se mantiene a través de transacciones complementarias con R.: Niña Adaptada Sumisa - Padre Crítico, que sitúan a J. en una situación de vulnerabilidad a la depresión. En relación con estas transacciones es importante mencionar también las leyes de economía de caricias, enumeradas al comienzo. Exceptuando la primera de ellas ("No dé las caricias positivas que corresponda dar"), el resto son aplicables a esta relación y, asimismo, coherentes con el rol asumido por J.

R., como Padre Crítico, defiende su sistema de economía de caricias con afirmaciones del tipo "No has hecho más que cumplir con tu obligación", "Es tu deber", "Para eso estás".

Respecto a los factores por los que $\mathbf{J}$. mantiene la asunción de rol, destacamos:

- El "temor" al conflicto y a que aparezca un estado emocional negativo en $R$. cuya responsabilidad emocional recaería sobre $\mathrm{J}$.

- Los sentimientos de "culpa" generados si no se cumplen exigencias que son planteadas como obligaciones asociadas a su rol.

- El "soborno" (las caricias son en su mayor parte condicionales, responden al cumplimiento del estereotipo).

- Las "descalificaciones" cuando J. plantea a R. necesidades y deseos o intenta que se afronte desde la pareja la crisis surgida.

Ante la imposibilidad de resolver en pareja la situación, J. opta por afrontarlo de manera individual buscando la creación de una nueva identidad que disminuya la ansiedad generada por el interrogante " ¿Quién voy a ser a partir de ahora?". Esto se plasma en el cambio hacia un rol de Adulta, que requiere también de $\mathbf{R}$. una modificación en su posición. La imposibilidad del cambio genera en J. sentimientos de ira y hostilidad hacia $\mathrm{R}$.

\section{Intervención}

\section{Objetivos}

- Redefinición de la identidad, para lo que se hace necesario:

- Aportar luz sobre aquellas características que $J$. posee pero a las que tanto su pareja como ella misma no atienden (darse "caricias positivas").

- Definirse en otras áreas vitales que no sean la doméstica.

- Mantener características nucleares flexibilizando y diferenciando los constructos "buena persona", "buena madre", "buena esposa" (Botella y Feixas, 1998).

- Aceptar las caricias positivas que merezca y pedir las que necesite, no sólo a su pareja sino también a otras personas.

- Rechazar las caricias negativas de R.

Con el cumplimiento de estos objetivos, según se observa en el modelo funcional, mejoraría el estado de ánimo y se reducirían los niveles de ansiedad, a la vez que se fomentaría una conducta más asertiva ante $R$.

Respecto a la emoción de ira, se prevé que el cambio hacia las leyes de abundancia de caricias establecidas con otras personas de su entorno reducirá la necesidad de un cambio en R. y, por tanto, dada la dificultad de este, la frustración y la ira.

\section{Técnicas}

Para el logro de los objetivos anteriormente mencionados, se utilizaron técnicas de distintos enfoques teóricos, enmarcadas siempre dentro del modelo explicativo del problema de J.

En un primer momento, la intervención se dirigió al aumento de actividades gratificantes, como una vía para el descubrimiento de aspectos de su identidad no explorados, la creación de una vida privada y el aumento de la red social (como forma de obtención de caricias positivas). $\mathrm{Al}$ mismo tiempo, fue necesario diferenciar los constructos "buena madre", "buena persona" y "buena esposa", así como buscar nuevos significados para ellos, de forma que J. se permitiera el 
desarrollo de estas actividades sin que estos constructos se vieran amenazados. Este inicio de reconstrucción de lä identidad redujo la pérdida que se había producido y mejoró, en consecuencia, el estado de ánimo. Durante todo el proceso de la terapia, $\mathbf{J}$. ha ido incorporando actividades a su vida, de forma que también la sintomatología anxiógena (¿quién voy ser a partir de ahora?) se redujera progresivamente.

A lo largo de toda la terapia, se fueron planteando con J. cuáles eran las características atribuidas a su rol femenino tradicional, así como al de su pareja. Esto le permitió comprender su propio comportamiento y el de R., sin culpabilizar a ninguna de las partes, pero demandando al mismo tiempo un cambio en las pautas de relación establecidas.

El uso del Inventario del Concepto de Sí Misma (McKay, 1991), junto a la planificación de actividades y el aumento de la red social, facilitó a J. la ampliación de su autoconcepto, de modo que este no quedara reducido a los constructos anteriormente mencionados. Al mismo tiempo, la técnica supuso la flexibilización del rol de género asumido, desde el que se hacía inviable el reconocimiento de aspectos positivos que no fueran los estrictamente relacionados con el papel de madreesposa.

De forma espontánea, como consecuencia de la redefinición de la identidad, J. interrumpió conductas asociadas a su rol, consideradas anteriormente como obligaciones.
Como estrategia para aprender a rechazar las caricias negativas de R. y afrontar las descalificaciones, se explicaron y entrenaron habilidades de comunicación a través de la representación de papeles.

El abordaje de la decisión (como YO Adulto) respecto a una posible separación se pospuso, debido a que J. vivía como una ruptura biográfica importante el divorcio y para que esta opción fuera considerada en la misma medida que otras se hacía necesario primero la creación de un espacio propio y un asentamiento de la identidad desde los que concebir esta posibilidad. La opción de establecer nuevas pautas de relación con $R$. fue inviable, puesto que este "descalificó" dicha propuesta de solución.

Finalmente J. se planteó la separación Dentro de este proceso, se hizo necesario nuevamente tomar una decisión, en este caso respecto a la búsqueda de empleo.

Durante la última fase de la terapia, surgió la dificultad de asumir, sin sentimientos de culpa, el haberse mantenido en esta situación durante los años de relación con $\mathrm{R}$.. Para resolverlo, se retomó la explicación, desde una perspectiva de género, de los comportamientos de ambos y J. elaboró una historia en tercera persona sobre el proceso vivido.

\section{Resultados}

SCL-90-R:

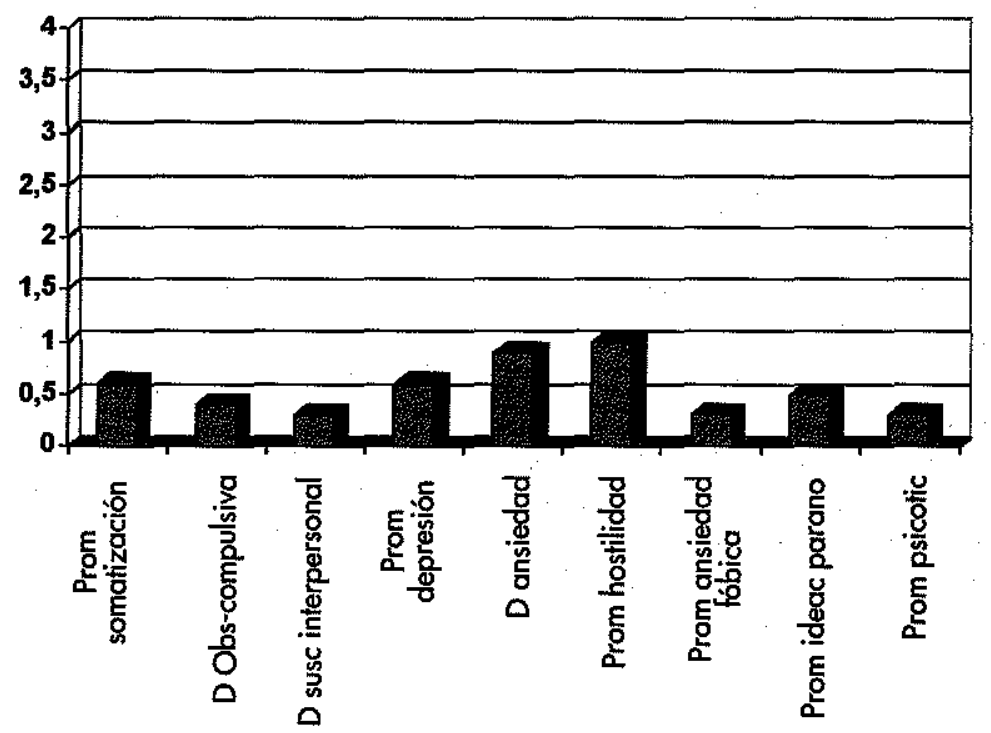


B.D.I

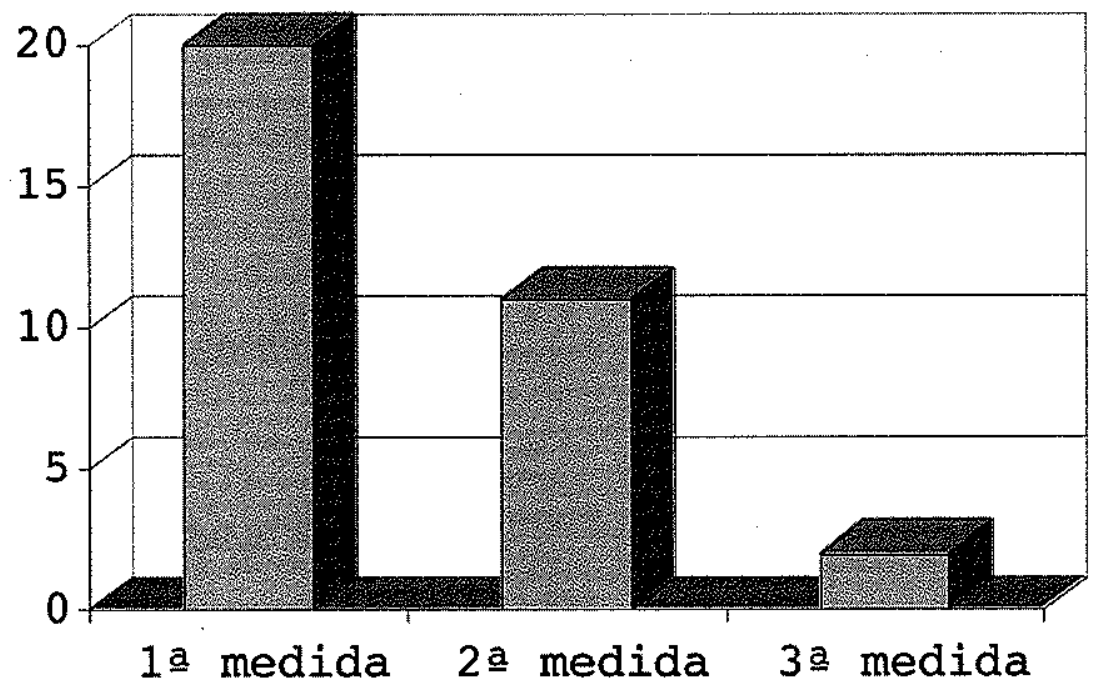

S.T.A.I.

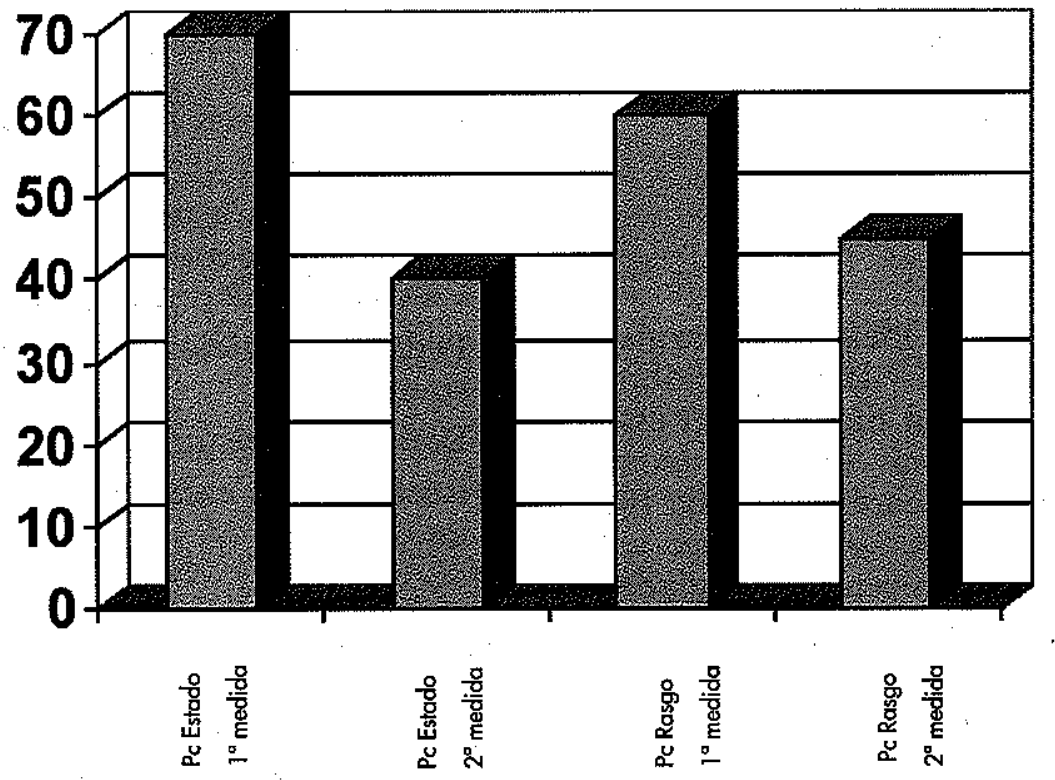

En un principio, el autoconcepto de J. se basaba en ser una buena persona, con valores de familia, responsable y trabajadora. Al finalizar la terapia, J. se define a sí misma a través de constructos referidos a distintas áreas vitales:
- Extrovertida-sociable

- Activa, con inquietudes

- Buena persona

- Buena madre

- Fuerte 


\section{Significados anteriores:}

Buena persona=Buena madre-esposa<smiles>c1ccccc1</smiles>

Portarse bien<smiles>C1CCCCC1</smiles>

Ceder en sus derechos<smiles>C1CCCCC1</smiles>

Dedicar tiempo a los demás<smiles>C=C</smiles>

Ofrecer ayuda antes de que te la pidan

\section{Significados actuales:}

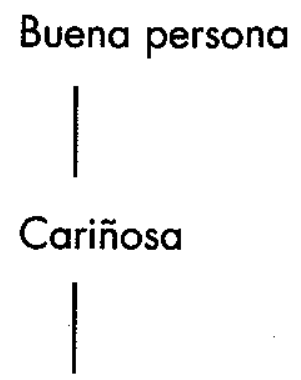

Que ayuda<smiles>C1CCCC1</smiles>

Que tiene opinión

Que defiende sus derechos

Figura 2. Constructos personales pre y post tratamiento

- Asertiva

- Decidida

- Adaptable, flexible

- Con valores de familia

Esta redefinición de la identidad, paralela al aumento de la red social y la realización de actividades gratificantes, ha mejorado el estado de ánimo y reducido los niveles de ansiedad. Se mantiene aspectos nucleares para ella (ser buena madre, ser buena persona y tener valores de familia), pero aparecen diferenciados. $J$. presenta una conducta asertiva ante $R$. y se plantea la ruptura de la relación cuando en unos meses el hijo que actualmente convive con la pareja se independice. Ha comentado en repetidas ocasiones la sensación de "haberse liberado de los sentimientos respecto a $R$." $y$ una nueva actitud resumida en "pensar en sí misma".

En términos de análisis transaccional, J. ha sustituido las Leyes de Economía de Caricias por las Leyes de Abundancia de Caricias:
- Dar a los demás las caricias adecuadas que necesitan y se merecen.

- Aceptar uno/a mismo/a las caricias adecuadas que se merece en honor a su persona y a sus actividades y conducta.

- Pedir las caricias adecuadas que se necesiten.

- Darse a sí mismo/a las caricias adecuadas.

- Rechazar las caricias inadecuadas que den los demás.

\section{Conclusiones}

El proceso de transformación de los roles de género que experimentamos en la actualidad supone un cambio que no se produce al mismo ritmo en todos los contextos y edades. Sabemos que en nuestro país la tasa de desempleo femenina es superior a la masculina y que aún existen diferencias en la remuneración económica por el mismo trabajo en función del género. Sin embargo, aunque encontramos aún que se mantienen 
las identidades tradicionales, muchas mujeres cuya socialización se ha visto determinada por los rasgos de género que se citaban al comienzo, han sustituido estos por otros más igualitarios. Este cambio implica necesariamente una modificación de la posición en la relación de pareja. Podemos observar mujeres que parecen haber vivido dos etapas claramente diferenciadas; una (la primera) basada en el cuidado hacia la familia y la negación de las propias necesidades y deseos, y otra (posterior) que implica una mayor independencia y autonomía. La transición de una a otra etapa conlleva una crisis vital, cuya resolución dependerá de factores personales, familiares y del entorno social más próximo. Pero tienen también una importancia fundamental elementos del macrosistema mencionado al inicio: desde la perspectiva de género se ha relacionado la estructura de la sociedad patriarcal con el sistema económico de los países industrializados. Parece imprescindible para el logro de una sociedad equitativa la generación de cambios estructurales, que reviertan en cambios en las relaciones de poder.

\section{Bibliografía}

Amorós, J. (dtora.) (1995). 10 palabras clave sobre mujer. Navarra. Ed.: EVD.

Berne, E. (1981). Juegos en que participamos: psicología de las relaciones humanas. México. Ed.: Diana.

Botella, L. y Feixas, G. (1998). Teoria de los constructos personales: aplicaciones a la práctica psicológica. Barcelona. Ed.: Laertes.

Bronfenbrenner, U. y Mahoney, M.A. (1975). Influences on human development. Illinois. Ed.: The Dryden Press.

Kertesz, R. y Induni G. (1978). Manual de Análisis Transaccional. Buenos Aires. Ed. Conantal.

MacKay, M. y Fanning, P. (1991). Autoestima: evaluación y mejora. Barcelona. Ed.: Martínez Roca.

Murillo, S. (1996). El mito de la vida privada. Madrid. Ed.: Siglo XXI.

Steiner, C. (1981). The Other Side of Power. New York. Ed.: Grove Press.

Tannen, D. (1996). Género y discurso. Barcelona. Ed.: Paidós. 